\title{
Semi-span wind tunnel testing without conventional peniche
}

\author{
S. N. Skinner ${ }^{1} \cdot$ H. Zare-Behtash ${ }^{1}$ (1)
}

Received: 3 May 2017 / Revised: 29 September 2017 / Accepted: 30 September 2017 / Published online: 8 November 2017

(C) The Author(s) 2017. This article is an open access publication

\begin{abstract}
Low-speed wind tunnel tests of a flexible wing semi-span model have been implemented in the $9 \times 7 \mathrm{ft}$ de Havilland wind tunnel at the University of Glasgow. The main objective of this investigation is to quantify the effect of removing the traditional peniche boundary layer spacer utilised in this type of testing. Removal of the peniche results in a stand-off gap between the wind tunnel wall and the model's symmetry plane. This offers the advantage of preventing the development of a horseshoe vortex in front of the model, at the peniche/wall juncture. The formation of the horseshoe vortex is known to influence the flow structures around the entire model and thus alters the model's aerodynamic behaviours. To determine the influence of the stand-off gap, several gap heights have been tested for a range of angles of attack at $R e=1.5 \times 10^{6}$, based on the wing mean aerodynamic chord (MAC). Force platform data have been used to evaluate aerodynamic coefficients, and how they vary with stand-off heights. Stereoscopic Particle Imaging Velocimetry (sPIV) was used to examine the interaction between the tunnel boundary layer and model's respective stand-off gap. In addition, clay and tuft surface visualisation enhanced the understanding of how local flow structures over the length of the fuselage vary with stand-off height and angle of attack. The presented results show that a stand-off gap of four-tofive times the displacement thickness of the tunnel wall boundary layer is capable of achieving a flow field around the model fuselage that is representative of what would be expected for an equivalent full-span model in free-air-this cannot be achieved with the application of a peniche.
\end{abstract}

H. Zare-Behtash

Hossein.Zare-Behtash@glasgow.ac.uk

1 School of Engineering, University of Glasgow, Glasgow G12 8QQ, Scotland, UK

\section{Introduction}

Semi-span testing techniques have been widely adopted as a tool to provide state-of-the-art wind tunnel research capabilities (Lynch 1992; Viehweger and Ewald 1994; Silva et al. 2012; Nguyen et al. 2015). Semi-span models are typically employed to double the maximum usable Reynolds number and enhance the quality of measurement data owing to improved model strength, stiffness, and overall fidelity (Millholen II and Chokani 1996). However, semi-span models also have inherent difficulties associated with the quality of flow over the model, and are vulnerable to increased interference effects within the wind tunnel. The basic principle of semi-span testing is to treat the mid-plane cross section of the body as a plane of symmetry. This is generally achieved by mounting the mid-plane of the body to some planar surface (such as the wind tunnel floor, wall, or ceiling) enabling the surface to act as a plane of symmetry. The wind tunnel walls, however, provide poor symmetry planes due to the growth of respective boundary layers which begin to develop far upstream of the model. Interactions between the working section boundary layer and the model can introduce threedimensional vortical structures (horseshoe vortices), which are recognised to have strong influences on the semi-span model's aerodynamic behaviours, especially at the wing root (Doerffer and Szulc 2006).

To reduce the aforementioned interactions with the tunnel boundary layer/wall, and achieve near free-air conditions, semi-span models traditionally use the peniche technique. The traditional peniche is a two-dimensional profile extrusion identical to the model's mid-plane cross section to create a stand-off distance between the mid-plane and the tunnel wall. The stand-off distance is typically scaled to the boundary layer displacement thickness $\left(\delta^{*}\right)$ at the wind tunnel wall at the pitch axis/moment reference centre of 
the model. Unfortunately, studies are generally ambiguous in quantifying the complex interactions between the wall boundary layer and the peniche, including the resulting effects and sensitivity on wing aerodynamics. In general, the peniche is scaled through a trial-and-error approach forcing the semi-span model to mimic the full-span model's aerodynamic coefficients for a small range of angles of attack at a given Reynolds number. Variations in peniche height are often seen to reflect variations in lift-curve slope, stall characteristics and body moments as local flow phenomena governed by peniche are not well understood.

Studies by NASA Langley (Millholen II et al. 1996; Gatlin and McGhee 1996; Gatlin et al. 2001) have reported that the influence of the peniche is to accelerate the flow over the entire upper surface of the wing, and simultaneously increase the cross flow observed over the inboard upper surface area of the wing. Conclusions suggest that a standoff height equal to twice the displacement thickness of the empty tunnel boundary layer works best to mitigate this, and that modifications by filleting or undercutting the peniche (a three-dimensional peniche) can alleviate separation, and the development of vortical structures around the fuselage leading nose. However, a standard 2-D peniche produced data which correlated better with full-span data (Gatlin et al. 2001).

Eliasson (2008) reports lower drag and maximum lift in semi-span wind tunnel experiments relative to the expected free-flight values. It was concluded that the discrepancy is a consequence of the peniche enforcing a redistribution of the velocity field and increasing cross-flow components in the plane of symmetry of the half model. The peniche's effect on the model enforces additional flow displacement leading to additional velocity gradients around the fuselage and inboard wing compared to a full-span model. Hence, the peniche is not able to supply a velocity field sufficiently parallel to the symmetry plane-especially at higher angles of incidence. Eliasson (2008) suggests that changing the peniche height only redistributes the strength of the flow displacement and cannot be avoided, but that the shape of the peniche may alleviate this. Other studies have tried to reduce the flow displacement, and resulting vortical structures, through leading edge suction, thereby reducing mass flow and velocity gradients (Malik and Render 2010). There is a little evidence to suggest, however, that suction ahead of the peniche can produce a significant improvement in the aerodynamic characteristics of semi-span models.

Doerffer and Szulc (2006) performed a numerical study of the wing-body configuration of the DLR F11 high-lift model to compare free-flight conditions to the semi-span model. Computations of the half-span model placed directly at the wall and mounted using a standard peniche (at approximately $3 \delta^{*}$ ) are presented. With the application of the peniche, the strength and size of the horseshoe vortex around the fuselage nose increased. It was found that the introduction of the peniche to the semi-span model led to poorer agreement with full-span data; this was based on comparisons between pressure distributions over the wing and aerodynamic coefficients $C_{\mathrm{L}}, C_{\mathrm{D}}$, and $C_{\mathrm{M}_{\mathrm{p} i c h}}$. While it is concluded that best results were found for the semi-span model mounted directly to the wall without the addition of a peniche, the authors speculate that another factor, other than the peniche, is responsible for the lift break-down of the DLR F11 half model in the experiment. No attempts were made to vary the stand-off height of the peniche.

Yokokawa et al. (2010) conducted a comprehensive experimental-numerical study focused on how the aerodynamic influence of a half span model changes with a peniche in an attempt to strategize the appropriate selection of the peniche stand-off height. While they observed no changes in surface flow patterns (using oil flow visualisations) with increasing peniche heights, strong changes in the aerodynamic coefficients and pressure distributions are noted. Lift-slope gradients were observed to increase with larger peniche height, and inconsistency of data for different peniche heights indicated changes in local flow physics over the wing. Furthermore, the drag at a given angle of attack decreases as the peniche height increases; however, they show that the inboard wing local drag increases. The pressure distribution over the wing shows that only the leading edge of the wing is significantly affected by changes in peniche height. Yokokawa et al. (2010) suggest that different peniche installations have the effect of changing the models effective aspect ratio, thus strongly influencing the induced drag. To test this hypothesis, they implement numerical simulations to predict optimal peniche heights to best resemble free flight conditions. It is found that two-to-three times the displacement thickness of the empty wind tunnel boundary layer is optimal for matching effective aspect ratios.

It is common to find studies which pay a little attention to the appropriate sizing of the peniche. Ujang et al. (2016) neglect investigation of the peniche's influence entirely, and opt to simply maximise the stand-off height in an attempt to raise the model out of the wall boundary layer. In doing so, the solid-body blockage of the model was significantly increased. It can also be expected that the vortical structures around the fuselage nose are not mitigated but in fact amplified as the peniche reaches into the free-stream. In a similar manner, Kafyeke et al. (2002) use a boundary layer spacer plate to isolate the model from the wall boundary layer. They conclude that the aerodynamic coefficients were comparable to flight test data; however, pitching moment data across most of the test range and post stall behaviour correlated poorly. Kuo, C and Lin (1996) demonstrated the application of a boundary layer spacer successfully integrated with a 
semi-span delta wing model. It was found that the boundary layer spacer reduced the influence of the tunnel wall boundary layer on the vortical structures over the wing. Elsewhere Lee and Su (2012), semi-span delta wing models have been mounted on very long slender 2-D peniche extrusions.

The application of the peniche attempts to reduce flow interference with the tunnel wall and achieve a flow field representative of the equivalent full-span model freeair. However, the introduction of the peniche appears to introduce more flow complexity and uncertainty to the measurements. A solution, or at least a simple alternative, is to remove the peniche entirely and leave a stand-off gap as suggested by Eder et al. (2006) The 2-D peniche and resulting vortical structures modify the behaviour of the model, this is difficult to quantify and correct for. Eder et al. (2006) show that the peniche height introduces varying discrepancies between full-span and semi-span aerodynamic coefficients and moments, and that these discrepancies vary with angle of attack. Deviations that are not constant for a range of angles of attack and Reynolds number suggest significant changes in the local flow physics over the model. Without a peniche, thus placing a gap between the fuselage and the wall, deviations in aerodynamic coefficients and moments were found independent of the angle of attack. A stand-off gap height of $4 \delta^{*}$ was found to achieve constant deviation of the aerodynamic coefficients from the equivalent full-span model over a range of angles of attack.

To date, there is no universally accepted method on how best to conduct semi-span testing despite the widespread use and recognised benefits of such models. A major drawback between different investigations is that it is difficult to draw comparisons of the various approaches adopted. Efforts are made here to investigate the effect that the stand-off gap height has on aerodynamic behaviours while also understanding the local flow around the semi-span model and how it changes with both stand-off gap height and angle of attack. The objective of the present experimental investigation is to vary the stand-off gap and examine the resulting interactions with the tunnel wall boundary layer and its aerodynamic influence over the semi-span model. In this study, no comparisons are made to any full-span models; thus, only experimental trends of varying the stand-off gap and its effects on the aerodynamic behaviour of the model are of primary interest. Flow around the fuselage nose is measured and visualised using stereoscopic particle imaging velocimetry (sPIV) and surface flow patterns and visual observations are obtained using tuft and clay flow visualisations. Aerodynamic coefficients are measured using a force platform with six degrees-of-freedom.

\section{Experimental set-up}

\subsection{Semi-span model}

The semi-span model, shown in Fig. 1, planform is analogous to a typical mid-sized transport commercial aircraft representing a generic 10\%-scale model. A modular design enables the outboard wing section to be freely changed connecting to the main wing section in such a way as to maintain a continuous flexural axis. To reduce complexity for both experimental and numerical campaigns, the wing is untwisted with a constant NACA 63-412 aerofoil section. A schematic diagram of the semi-span of the model used in this study is shown in Fig. 2, with additional specifications listed in Table 1. Re based on MAC with atmospheric properties taken from Anderson (2001).

The model is constructed from Polyurethane foam of varying densities to achieve the desired aeroelastic behaviour. The pitch axis can be adjusted over a range of $210 \mathrm{~mm}$. This accommodates adjustments to the pitch axis of the model relative to the force platform, if large changes in either the aerodynamic centre or the centre of gravity are created. This is necessary, as the lack of the peniche to support the model means, the configuration must be carefully balanced around the pitch axis. However, an advantage of eliminating the peniche extrusion, here, is that the complexity of integrating the semi-span model with the wind tunnel turn table and force platform is significantly reduced.

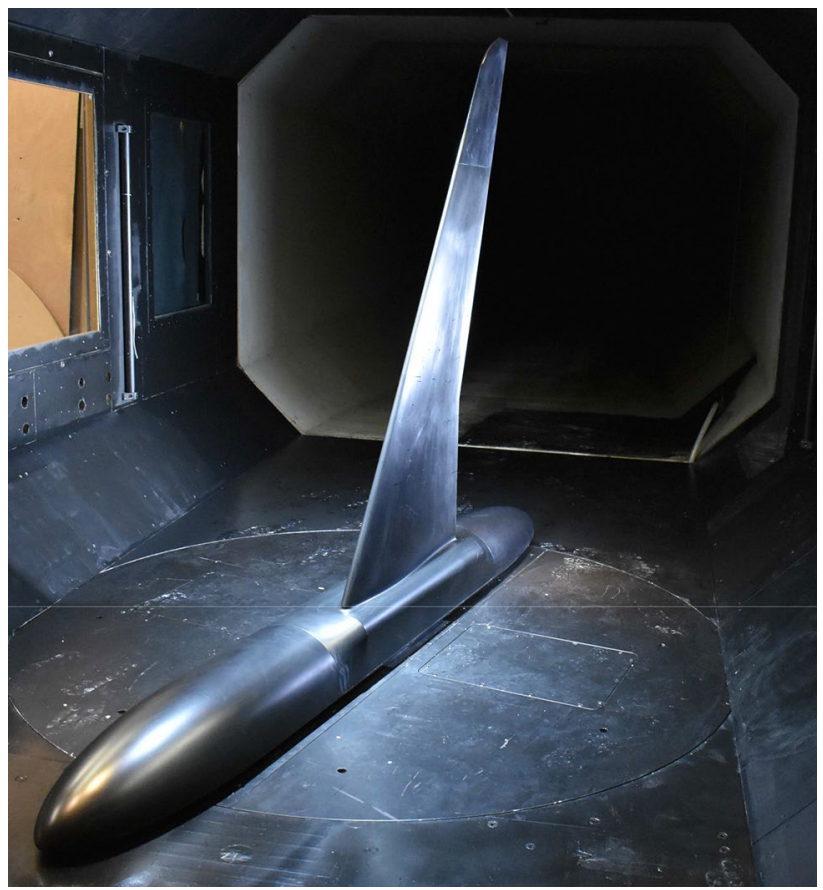

Fig. 1 Semi-span model installed in $\mathrm{dH}$ working section 
Fig. 2 Schematic of semi-span model; dimensions are in $\mathrm{mm}$

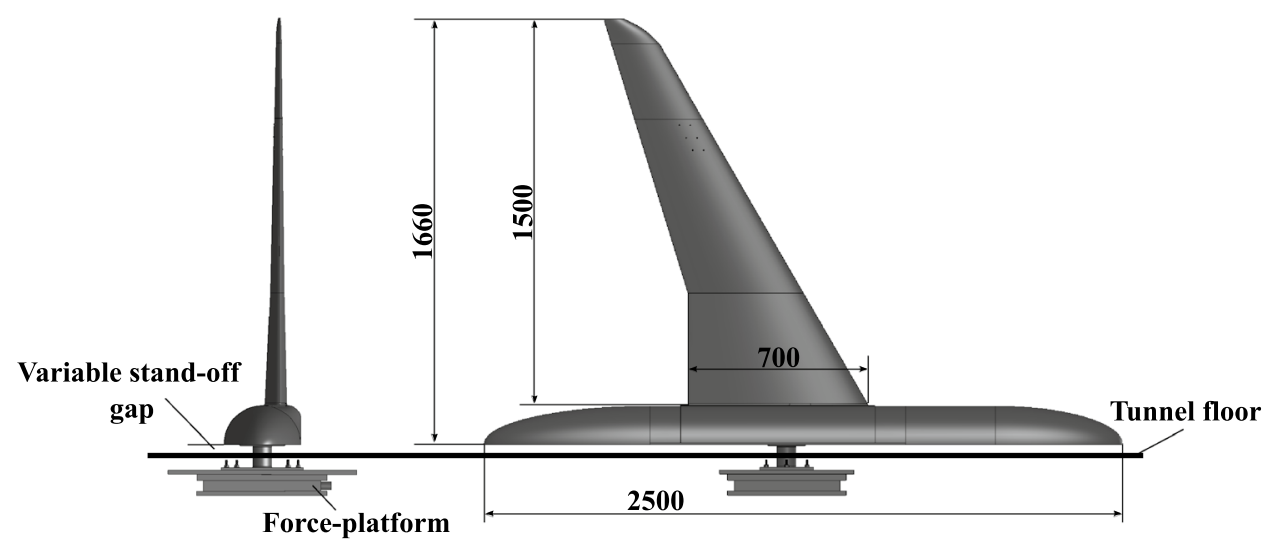

Table 1 Model parameters

\begin{tabular}{lll}
\hline & Typical full-scale & Semi-span model \\
\hline Mach number $\left(M_{\infty}\right)$ & $\approx 0.78-0.8$ & 0.145 \\
Reynolds number $(R e)$ & $\approx 10^{9}$ & $1.5 \times 10^{6}$ \\
Lift coefficient $\left(C_{\mathrm{L}}\right)$ & 0.5 & 0.5 \\
Altitude $(h)[\mathrm{m}]$ & $\approx 10,972.8(36,000 \mathrm{ft})$ & 0 \\
Dynamic pressure $\left(q_{\infty}\right)$ & $\approx 10,107.04$ & 1531.25 \\
$\quad[\mathrm{~Pa}]$ & & \\
Wing area $(S)\left[\mathrm{m}^{2}\right]$ & $\approx 65-90$ & 0.5717 \\
MAC $(\bar{c})[\mathrm{m}]$ & $\approx 4-5$ & 0.44 \\
Wing span $(b)[\mathrm{m}]$ & $\approx 17-19$ & 1.50 \\
Taper ratio $(\lambda)$ & $\approx 0.2$ & 0.21 \\
Aspect ratio $(\mathrm{AR})$ & $\approx 4-4.5$ & 4.12 \\
\hline
\end{tabular}

\subsection{Experimental facility}

The de Havilland wind tunnel $(\mathrm{dH})$ is an atmospheric lowspeed closed return wind tunnel circuit capable of reaching a Reynolds number of $4.8 \times 10^{6}$ per metre $\left(\approx 70 \mathrm{~ms}^{-1}\right)$. The working section has an octagonal cross section of $2.65 \mathrm{~m}$ width, $2.04 \mathrm{~m}$ height, and $5.64 \mathrm{~m}$ length with a contraction ratio of 5:1. The fillets are arranged to enable the test section's cross-sectional area to expand from inlet to outlet compensating for boundary layer growth and offset the resulting longitudinal static pressure gradients which contribute to horizontal buoyancy effects.

Knowledge of boundary layer growth through the test section is necessary for appropriate scaling of the standoff gap between the model and the tunnel. The boundary layer thickness $(\delta)$ growth rate through the test section is approximately linear, as shown in Fig. 3; data have been normalised by the working section length, $L$. Benchmark boundary layer data at several stations through the wind

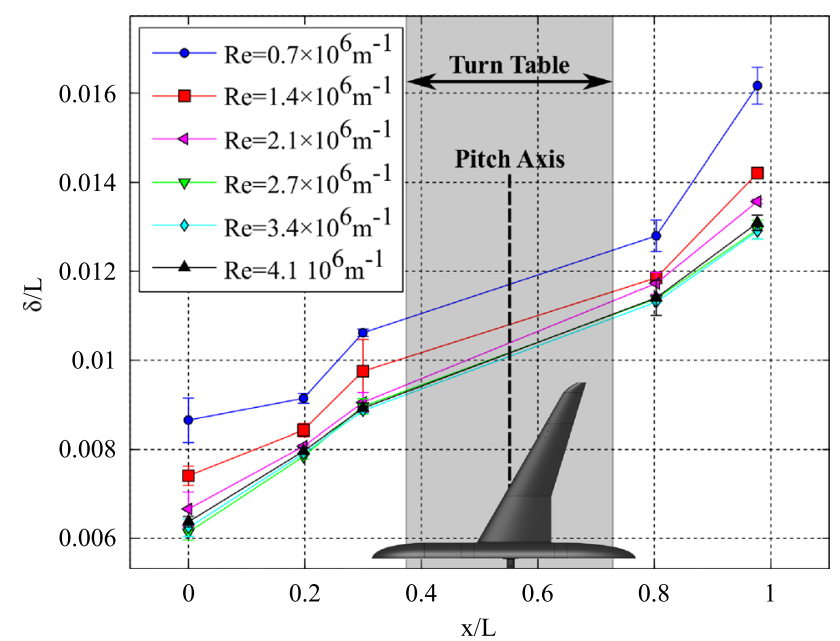

Fig. 3 Boundary layer thickness $(\delta)$, at different Reynolds numbers, through working section normalised by the $\mathrm{dH}$ working section length $(L)$

tunnel working section were sampled using a variable height pressure rake connected to a Scanivalve Corporation temperature compensated electronic pressure scanning module. The system consisted of an ERAD4000 A/D base with 16-bit resolution and two module connections, a ZOC22b pressure scanner ( 32 channels, \pm 1 psi range), and a ZOC23b pressure scanner (32 channels, \pm 10 inches $\mathrm{H}_{2} \mathrm{O}$ range) sampling at $250 \mathrm{~Hz}$ per channel for $20 \mathrm{~s}$. Figure 3 additionally suggests that the turntable positioning system does not significantly influenced the tunnel boundary layer.

The tunnel Ate AEROTECH 2 m-diameter turntable was used for model positioning, capable of providing $360^{\circ}$ motion at $5^{\circ} \mathrm{s}^{-1}$. The yaw of the turntable was used to control the angle of incidence of the model with a position accuracy is $\pm 0.005^{\circ}$. Aerodynamic coefficients have been assessed 
using an AMTI OR6-7 1000 series force platform which was housed in the centre of the turntable beneath the tunnel floor. The force platform measures the three orthogonal force components along the $X, Y$, and $Z$ axes, and the resulting moments about each axis. Measurement accuracy is $\pm 0.25 \%$ of the applied load on the respective output. Each output was recorded at $2 \mathrm{kHz}$ over $20 \mathrm{~s}$ for static conditions only.

For collaborative examination of dynamic loading recorded by the force platform, a Polytec PDV-100 digital laser vibrometer was employed for non-contact single degree of freedom velocity measurements up to $22 \mathrm{kHz}$. The point vibrometer made it possible to isolate certain frequencies and their sources; measurements were made over $26 \mathrm{~s}$ at a sampling frequency of $10 \mathrm{kHz}$, achieving a resolution of $39 \mathrm{mHz}$, with no filtering applied. As the vibrometer only provided data at a single position phased vibration, the wing torsional modes cannot be assessed.

Stereoscopic particle imaging velocimetry (sPIV) was performed to examine the flow-structure interactions between the tunnel boundary layer and the model fuselage nose. Optical access was provided through large optical grade glass windows on the port and starboard walls, as well as several optical access points on the roof of the working section. The flow was homogeneously seeded with olive oil particles with a maximum diameter of $0.9 \mu \mathrm{m}$, produced using a Pivtec-GmbH large seeding device with 160 high precision Laskin nozzles; seeding particles were introduced downstream of test section. Suitable particle diameter and density of seeding is required to capture the flow-tracing accurately. The flow-tracing capability of particles of diameter $d_{\mathrm{p}}$ and density $\rho_{\mathrm{p}}$ is quantified through the particle relaxation time $\tau_{\mathrm{p}}$. The theoretical behaviour for small spherical particles may be reduced to the modified Stokes drag law (Scarano 2008). The particle dynamic effects may be quantified by the Stokes number:

$S k=\frac{\tau_{\mathrm{p}}}{\tau_{\mathrm{f}}}$,

where $\tau_{\mathrm{p}}$ and $\tau_{\mathrm{f}}$ are the particle relaxation time and characteristic time scale, respectively:

$\tau_{\mathrm{p}}=\frac{d_{p}^{2} \rho_{\mathrm{p}}}{18 \mu_{\mathrm{f}}}$

$\tau \mathrm{f}=10 \frac{\delta_{\mathrm{LE}}}{\Delta V}$

For confidence in accurate flow tracing, $S k<<1$ has to be satisfied. $\delta_{L E}$ is the boundary layer thickness at the leading edge of the fuselage. Table 2 shows the time scales and Stokes number; $S k<<1$ is satisfied.
Table 2 Capability of seeding particles for flow-tracing

\begin{tabular}{llll}
\hline$\delta_{\mathrm{LE}}[\mathrm{mm}]$ & $\tau_{\mathrm{p}}[\mu \mathrm{s}]$ & $\tau_{\mathrm{f}}[\mu \mathrm{s}]$ & $S k$ \\
\hline 50.3 & 2.48 & 125 & 0.01984 \\
\hline
\end{tabular}

The flow is illuminated by a double-pulse Nd:YAG laser (wavelength of $532 \mathrm{~nm}$ ) with an energy output of $100 \mathrm{~mJ}$ per pulse. Pulse duration of the laser sheet is $8 \mathrm{~ns}$ with a uniform light-sheet thickness of $1.5 \mathrm{~mm}$ in this study. Figure 4 illustrates a schematic of the sPIV set-up with the laser sheet illuminating the centre line of the fuselage nose with camera 1 and camera 2 on the port and starboard of the working section, respectively. The laser was pulsed at $\Delta t=45 \mu \mathrm{s}$ intervals between two consecutive images, so that sufficient displacement for the tracer particles was achieved in both the boundary layer and the free-stream. Keane and Adrian (1992) showed that to achieve valid detection probability of at least $90 \%$, the image density (particles per interrogation window) must be greater than 15 particles with displacements covering $30 \%$ of the interrogation window. In this way, it is possible to link $\Delta t$ with the interrogation window dimension, $d_{\mathrm{I}}$, and pre-knowledge of the flow (i.e., maximum in-plane velocity, $\max \left|U_{\text {in-plane }}\right|$ :

$\Delta t<\frac{0.3 d_{\mathrm{I}}}{\max \left|U_{\text {in-plane }}\right|}$.

Phantom v341 cameras with a resolution of $2560 \times 1600$ were used to record the scattered light reflected from the seeding particles at $200 \mathrm{fps}$. The recorded images were initially divided into $32 \times 32$ pixels interrogation windows, and then, interrogation windows were refined to $16 \times 16$ pixels. Stereo cross-correlation analysis was performed using commercial PIV software (DaVis 8.0, LaVision) from which data were taken and post-processed (including time averaging of the sPIV data) using in-house MATLAB scripts. With this arrangement, two adjacent velocity vectors were separated by $1.7 \mathrm{~mm}$.

Local flow patterns on the tunnel floor and the model were visualised using tufts and clay flow visualisation. $30 \mathrm{~mm}$ tufts were applied to the fuselage, inboard wing/ wing root, and floor with a spatial resolution of $30 \mathrm{~mm}$ longitudinally by $15 \mathrm{~mm}$ laterally. Movement of the tufts were recorded continuously at $100 \mathrm{fps}$ over $10 \mathrm{~s}$ using the Phantom v341 camera. The model angle of attack was held constant during recording. The clay flow formulation consisted of fine white Kaolin clay suspended in paraffin. The clay mixture was placed upstream of the model on the tunnel floor, over the nose of the fuselage, and along the leading edge of the wing. The incident angle of the model was held constant with the tunnel running continuously until the paraffin had completely vaporised and the clay was dry. Colour 
Fig. 4 sPIV set-up in the dH working section

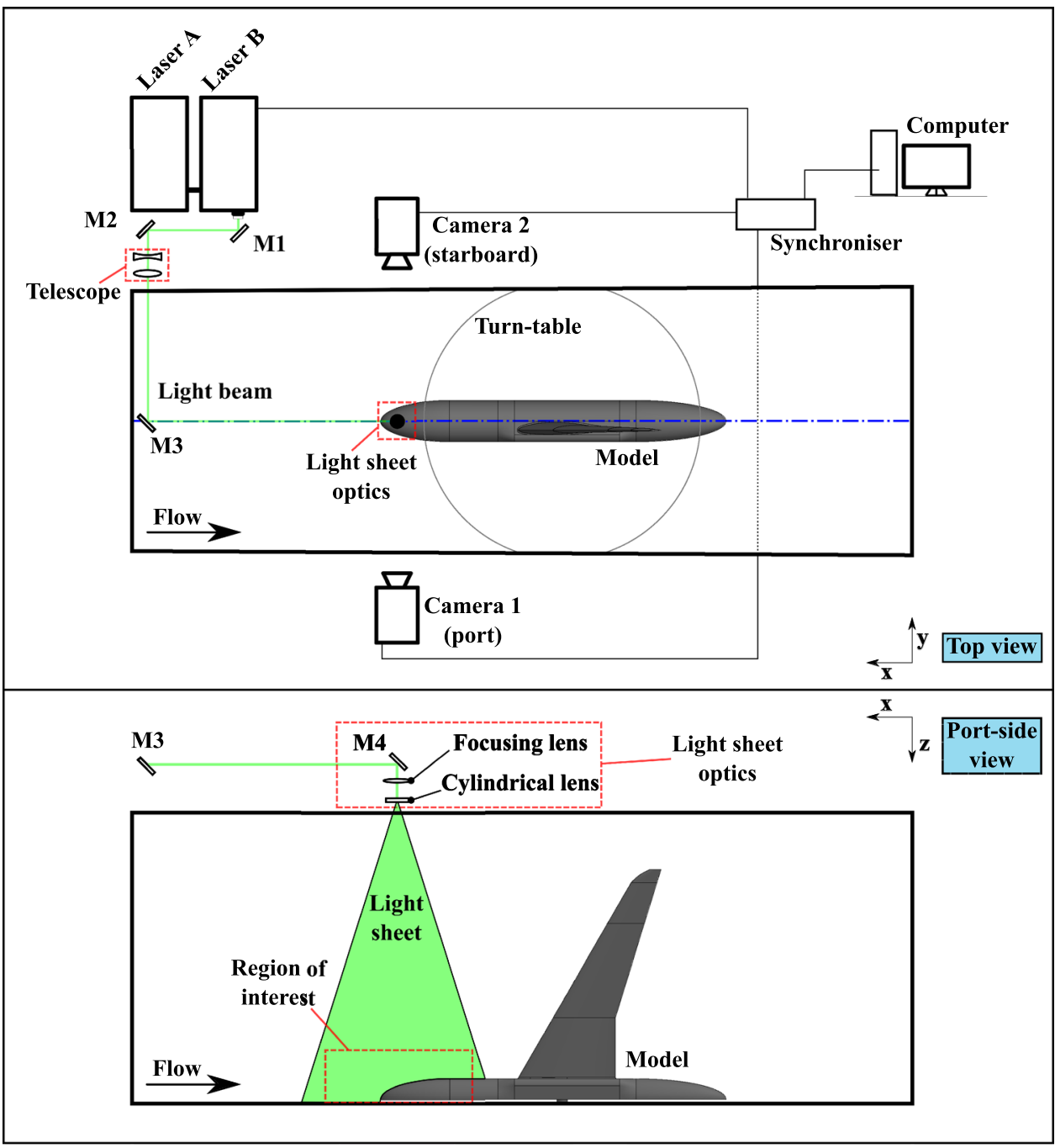

images were taken using a Canon SLR camera, model EOS450D with 12 Mega-pixel resolution.

\section{Results and discussion}

Data for different stand-off gap heights have been acquired for static angles of attack. All experiments were conducted at a Reynolds number of $1.5 \times 10^{6}$ based on the wing's mean aerodynamic chord, unless specified otherwise.

\subsection{Force and moment measurements}

Changes in lift, drag, and pitching moment about the model's centre of gravity/pitch axis are presented in Fig. 5, illustrating the behaviour of the aerodynamic coefficients with standoff gap height and angle of attack. All measurements have been corrected for solid-body blockage ( $4.9 \%$ at $\alpha=14^{\circ}$ ), wake blockage, and horizontal buoyancy using methods given by Barlow et al. (1999). The force and moment data presented are calculated with a confidence level of $98 \%$ and a margin of error less than $1 \%$ for each datum. The model has only been operated in the pre-stall region.

At all five stand-off heights, the lift coefficient, shown in Fig. $5 \mathrm{a}$, is linear between $-5^{\circ}$ and $+7^{\circ}$, with the maximum lift for each occurring at $+14^{\circ}$. The gradient $\left(C_{\mathrm{L}_{\alpha}}\right)$ decreases with increasing gap size. This trend was identified to be independent of Reynolds number, as shown by data presented in Fig. 6. The reducing gradient is primarily caused by increased mass flow though the gap between the tunnel wall and the model. This flow adds additional circulation around the fuselage which increases the induced angle of attack at the wing; this effect is illustrated in Fig. 7.

Figure 8a shows the deviation of the lift coefficient as a function of the angle of attack, taking the $1.4 \delta^{*}$ case as the baseline. While the general trend of $\Delta C_{\mathrm{L}}$ with angle of attack is consistent for each case, the deviation from the baseline indicates no definitive trend. An approximately constant offset exists for the angle of attack range $0^{\circ} \leq \alpha \leq+10^{\circ}$ for all cases, with a consist decrease in the 


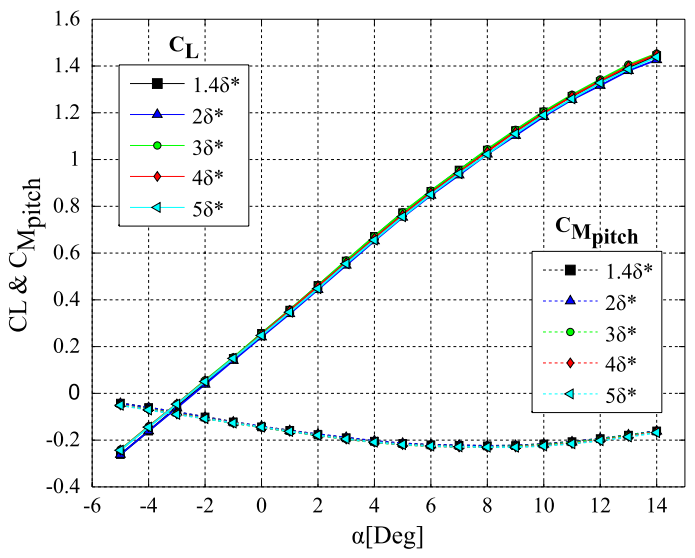

(a) $C_{L}$ vs. $\alpha$ \& $C_{M_{\text {pitch }}}$ vs. $\alpha$

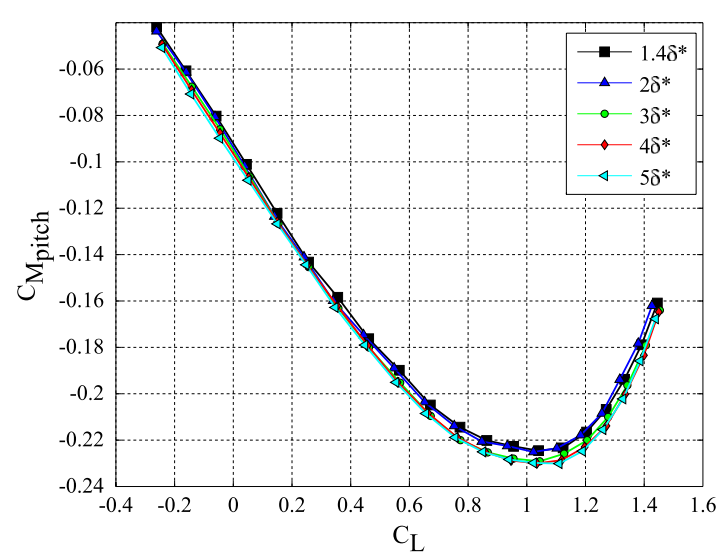

(c) $C_{M_{\text {pitch }}}$ vs. $C_{L}$

Fig. 5 Effect of stand-off gap on aerodynamic coefficients



Fig. 6 Lift gradient as a function of the stand-off gap height

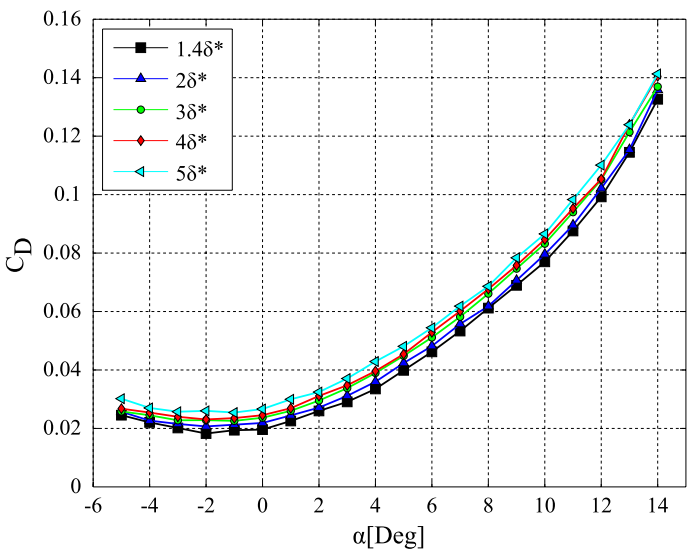

(b) $C_{D}$ vs. $\alpha$

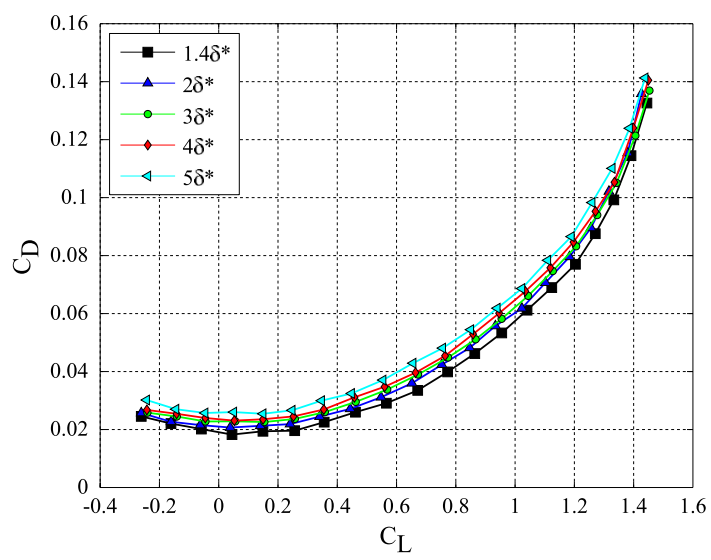

(d) $C_{D}$ vs. $C_{L}$

lift coefficient within this range from $3 \delta^{*}$ to $5 \delta^{*}$. Here, the deviation of lift coefficient is a function of the distance between the fuselage symmetry plane and the tunnel wall, and not a direct function of angle of attack. For $\alpha<0$ and $\alpha>+10$, the lift coefficient is a function of the angle of attack as well as the gap height; both instances are a direct effect of the shaft wake. At negative angles, the low-wing model configuration blocks incoming flow, such that the shaft make moves further over the fuselage afterbody than for equivalent positive angles of attack. With high angles of attack, the shaft wake spreads more to the upper side of the fuselage. The influence of the shaft wake is less for smaller stand-off gap heights.

Figure $8 \mathrm{~b}$ shows the deviation of the drag coefficient as a function of the angle of attack, taking the $1.4 \delta^{*}$ case as the baseline. It is clearly shown that with larger stand-off gap heights, the drag coefficient increases. Between angles of attack $-5^{\circ} \leq \alpha \leq+7^{\circ}$ (the linear portion of the lift curve), the difference in drag coefficient is almost a constant offset 
Fig. 7 Additional circulation around fuselage with larger stand-off gap (image to scale)

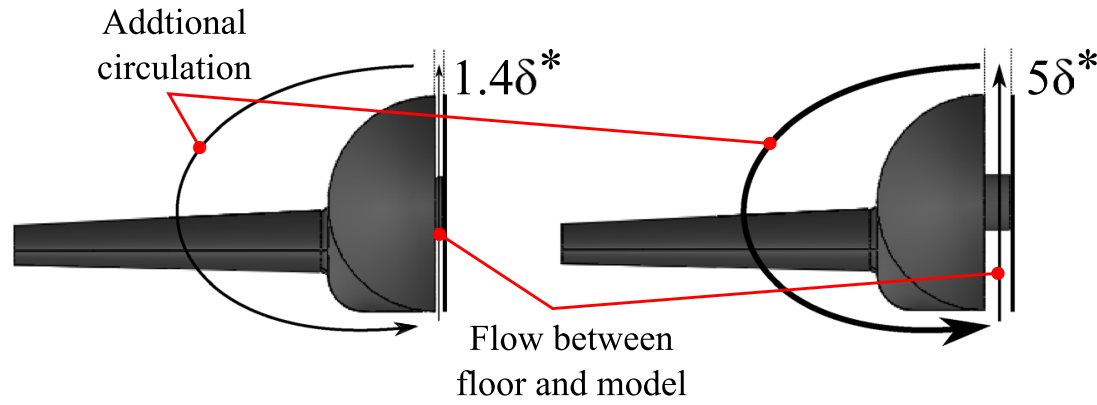

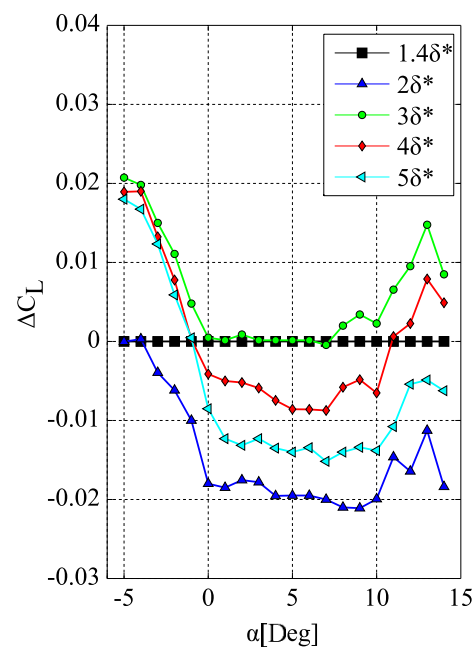

(a) $\Delta C_{L}$ vs. $\alpha$

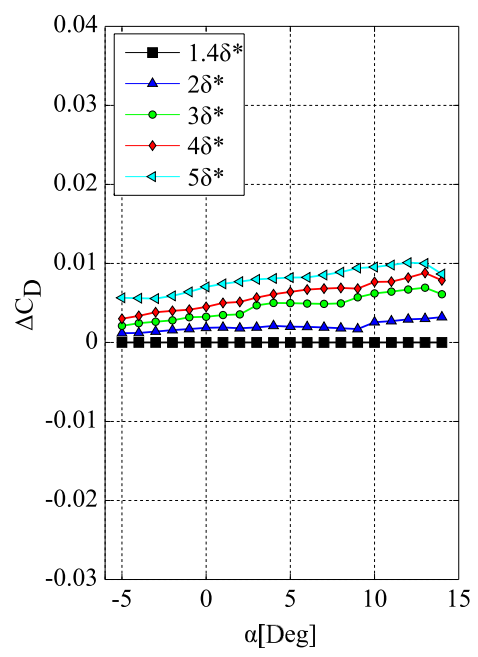

(b) $\Delta C_{D}$ vs. $\alpha$

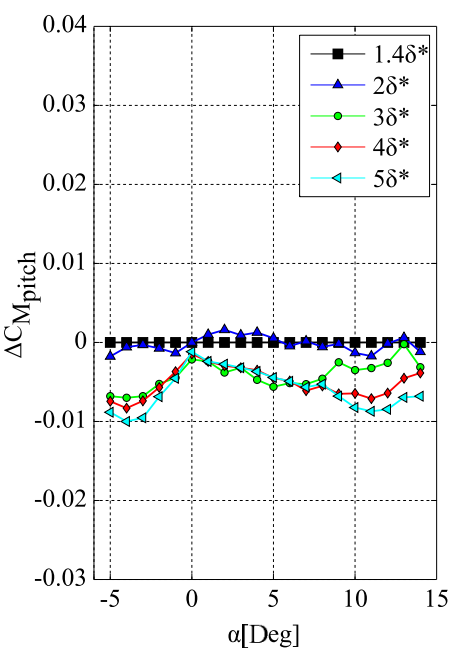

(c) $\Delta C_{M_{p i t c h}}$ vs. $\alpha$.

Fig. 8 Difference in aerodynamic coefficients taking stand-off gap height $1.4 \delta^{*}$ as baseline case

Table 3 Average drag coefficient deviation from $1.4 \delta^{*}$ baseline with stand-off gap over range $-5^{\circ} \leq \alpha \leq+7^{\circ}$; corresponding to data shown in Fig. 8

\begin{tabular}{ll}
\hline Test case & $\Delta C_{\mathrm{D}}$ offset \\
\hline $1.4 \delta^{*}$ & - \\
$2 \delta^{*}$ & +0.0017 \\
$3 \delta^{*}$ & +0.0038 \\
$4 \delta^{*}$ & +0.0051 \\
$5 \delta^{*}$ & +0.0072 \\
\hline
\end{tabular}

from the baseline case, where this offset increases with gap height. These $\Delta C_{\mathrm{D}}$ offsets have been summarised in Table 3 .

Here, the deviation in the drag coefficient is a function of the distance between the fuselage symmetry plane and the tunnel wall, and not a direct function of angle of attack. At angles of attack $\alpha>+7^{\circ}$, with the onset of wing separation, the deviation in the drag coefficient remains mostly constant, but slight variation with angle of attack is noticed.

The drag increase between the different stand-off heights is not associated with variations in the induced drag, but only those of the parasitic drag. The main increase of the drag is caused by flow past the symmetry plane of the fuselage. As the gap height increases, as illustrated in Fig. 7, the flow into the gap increases. The symmetry plane of the model, which is connected to the fuselage, will thus experience higher frictional forces. In addition, with increasing gap height, the shaft beneath the model incrementally extends, increasing the shaft's wetted area. Despite the disadvantage of the increased drag, the deviation of the drag coefficient is not a function of the angle of attack, as it would be with a peniche.

Figure $8 \mathrm{c}$ shows the deviation of the pitching moment coefficient as a function of the angle of attack from the baseline case, $1.4 \delta^{*}$. The deviation in the pitching moment for the $2 \delta^{*}$ stand-off is very small; however, at higher standoff gap heights, larger deviations which are a function of angle of attack occur. A significant decrease in the moment coefficient occurs between $2 \delta^{*}$ and $3 \delta^{*}$, indicating the aft movement of the aerodynamic centre. Stand-off heights $3 \delta^{*}$, $4 \delta^{*}$, and $5 \delta^{*}$ demonstrate similar behaviour (i.e., no further shifts of the aerodynamic centre) over the linear portion of the lift curve $\left(-5^{\circ} \leq \alpha \leq+7^{\circ}\right)$. The pitching moment coefficient is particularly stable over the range $0^{\circ} \leq \alpha \leq+7^{\circ}$. At 
higher angles of attack $(\alpha>+7)$, as stall onset over the wing spreads, the deviations vary more strongly.

\subsection{Vibration tests}

Semi-span wind tunnel testing without a peniche supporting the model may facilitate increased vulnerability to forced vibration due to fewer points of structural support. However, removal of the peniche eliminates aerodynamic complexities (horseshoe vortex) as previously discussed, and additionally

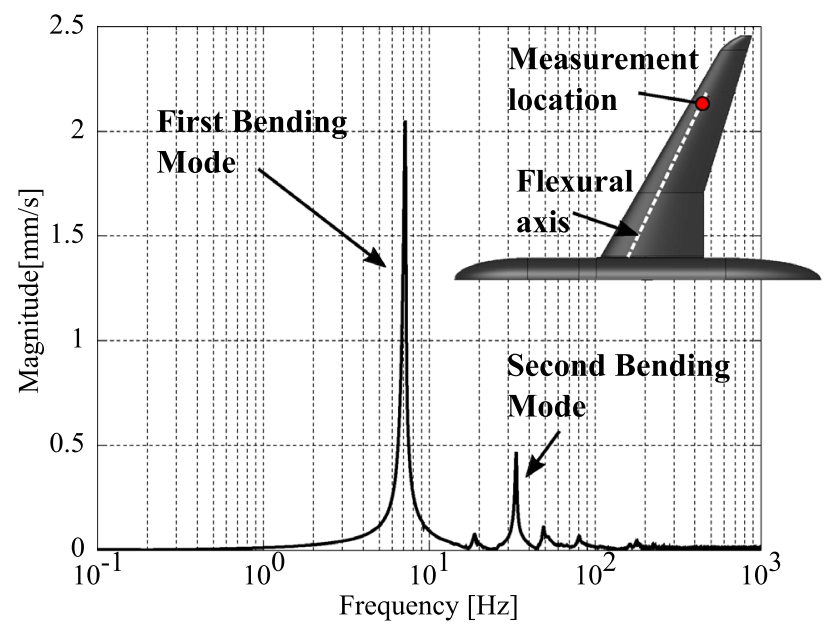

(a) Wing bump test with laser vibrometer (no airflow). makes mounting the semi-span to the force platform far simpler. A single mounting point at the pitch axis connects the model to the force platform; peniche-based semi-span models are supported across the entire plane of symmetry.

Dynamic loading on the model warrants examination due to the aeroelastic nature of the wing. Frequencies of the model detected from bump tests (with no airflow) and wind tunnel experiments are shown in Fig. 9; here, all data were recorded with the model mounted at $5 \delta^{*}$. The model does not change in any physical/structural way between different

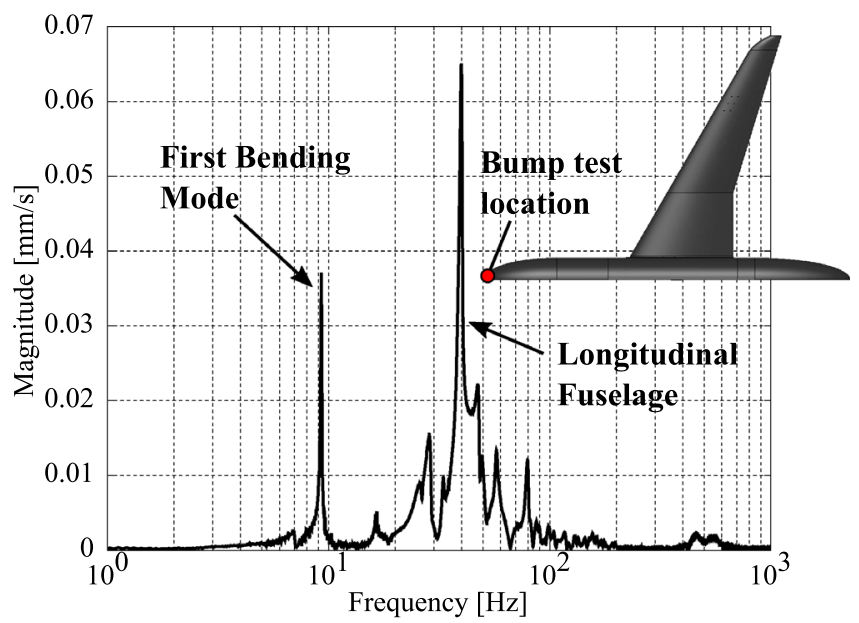

(b) Longitudinal fuselage bump test with laser vibrometer (no airflow).

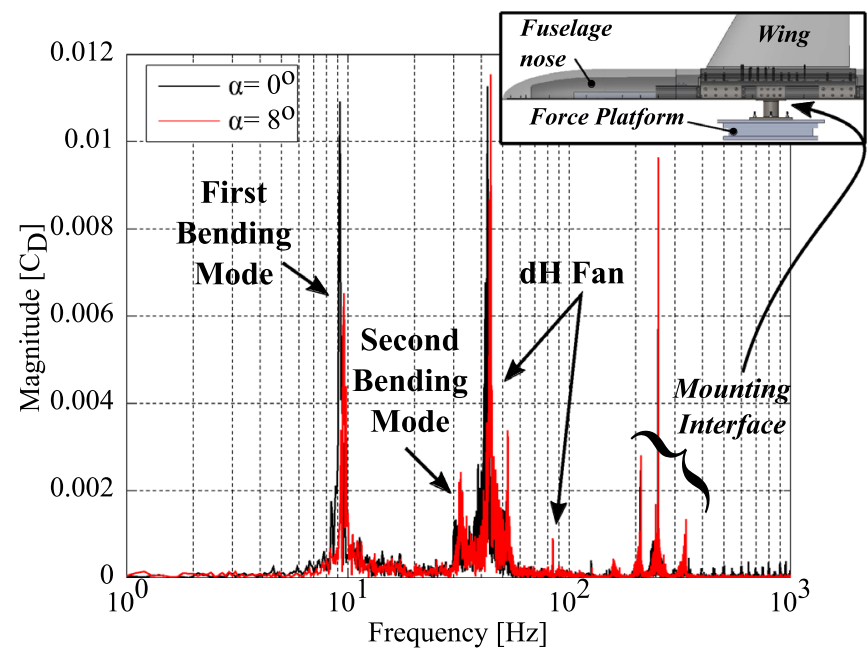

(c) Drag dynamic loading frequencies evaluated from force platform at $R e=1.5 \times 10^{6}$ for $5 \delta^{*}$ at $\alpha=0$, and 8 .

Fig. 9 Frequencies of the model observed in bump tests (bump test locations indicated by red dot in respective figures) and for wind tunnel tests 
stand-off gaps, and hence, vibrational frequencies present within the model will not change with stand-off gap height.

Results from the single point laser vibrometer used for bump tests on the wing and fuselage are shown in Fig. 9a, b, respectively - red points indicated on the model schematic in each figure show the bump test measurement location. This enabled the isolation of certain natural modes of the model. For the wing bump test (Fig. 9a), two bending modes are identified; the first mode of the wing is also detected in the fuselage longitudinal axis bump test shown in Fig. 9b. In Fig. 9b, dominant frequency detected for the longitudinal vibration of the model fuselage is shown at $40 \mathrm{~Hz}$.

Fourier transformations of the data gathered by the force platform enable the examination of frequencies present within the live experiments; bump tests enabled sources of vibrational modes to be identified. The Fourier transformation of the drag dynamic loading is shown in Fig. 9c. Both the first and second wing bending modes are visible; however, other dominant frequencies are clearly present which were not detected in bump testing. These additional frequencies are forced vibrations acting on the model arising from the wind tunnel interacting with the model, some of which are found to excite natural frequencies of the model. Frequencies and sources, which are shown in Fig. 9, are summarised in Table 4.

The operational settings of the wind tunnel fan are found to excite the longitudinal frequency of the fuselage giving explanation to the dominant $40 \mathrm{~Hz}$ frequency shown in Fig. 9c. To achieve the test condition of $R e=1.5 \times 10^{6}$ in the working section, the wind tunnel four bladed fan rotated at $600 \mathrm{rpm}(10 \mathrm{~Hz})$. The eight stators in the fan section result in a $80 \mathrm{~Hz}$ blade passing frequency, which, in part, would also be a harmonic of the fan blades' $40 \mathrm{~Hz}$. In addition, frequencies arising from steel mounting shaft and internal support structure inside the fuselage are visible the frequency analysis. Although the vibration tests provide wind tunnel and model specific information, results emphasise that mounting interfaces of this type can introduce loading frequencies from interactions between the facility and the model. While the support is stiff enough to prevent any movement of the model, it does connect the semi-span model directly

Table 4 Summary of frequencies detected and source

\begin{tabular}{ll}
\hline Source & Frequency $[\mathrm{Hz}]$ \\
\hline First wing bending mode & 7.188 \\
Second wing bending mode & 33.516 \\
Longitudinal fuselage mode & 39.940 \\
dH fan at 600 rpm (four blades) & 40.003 \\
dH Fan (eight stators) & 80.007 \\
Fuselage mounting interface (including shaft) & $211.1,253.2,337.6$ \\
\hline
\end{tabular}

to the force platform, such that any/all structural vibrations are detected.

\subsection{Stereoscopic particle imaging velocimetry (sPIV)}

The time-averaged flow fields showing normalised velocity magnitude and streamlines for stand-off heights of $1.4 \delta^{*}$, $3 \delta^{*}$, and $5 \delta^{*}$ are presented in Fig. 10. The dashed box in the top row of images illustrates the location of the lower images which provide an enhanced view of the flow around the model nose for each test case shown. The flow field shown is taken from the centreline of the fuselage nose at $\alpha=0^{\circ}$. The spatial co-ordinates have been normalised by the displacement thickness $\left(\delta^{*}\right)$ of the empty wing tunnel boundary layer at the moment reference centre (pitch axis) of the model, as shown in Fig. 3.

Results presented in Fig. 10 show no formation of a horseshoe vortex at any gap height. Thus, with the absence of the peniche, a more stable flow field is achieved. In addition, as the stand-off height increases, the velocity gradients around the stagnation point at the fuselage nose become more axi-symmetric. Considering the streamlines illustrated indicating the local flow, it is possible to qualitatively assess the effect that changing the stand-off gap height has on the incoming flow/respective boundary layer. At $1.4 \delta^{*}$ stand-off height, shown in Fig. 10a, the streamlines in the boundary layer are deflected upwards as they approach the model, lifting of the tunnel floor boundary layer. Increasing the standoff gap alleviates this effect as more of the boundary layer is drawn into the gap under the model, as shown in Fig. 10b, c. At $5 \delta^{*}$ (shown in Fig. 10c), the flow field around the symmetry plane of the model begins to closely resemble what could be expected for an equivalent full-span configuration at the symmetry plane. The flow near the tunnel wall is accelerated under the model, evident from the increase in velocity, and hence, the flow does not separate away from the wall and no horseshoe vortex is formed. The streamlines above the symmetry plane of the model remain undeflected. Thus, as the stand-off height increases, the peak velocity deficit corresponding to the centre of the stagnation point moves closer to the symmetry plane.

Normalised velocity profiles of the boundary layer at stream-wise stations $x / \delta^{*}=-20, x / \delta^{*}=-10, x / \delta^{*}=-5$, and $x / \delta^{*}=-0.5$ are presented in Fig. 11; the empty tunnel benchmark boundary layer at this station is also shown. At $x / \delta^{*}=-20$, Fig. 11a, the velocity profiles at all stand-off conditions are similar to the empty tunnel velocity profile. At this station, there is a quantity deficit in the $V_{x}$ component due to the presence of the model. Increases in the $V_{y}$ component at this station are of the order of $3 \%$. The $V_{y}$ increase is stronger for lower gap heights due to the lifting of the boundary layer. 




(a) $1.4 \delta^{*}$
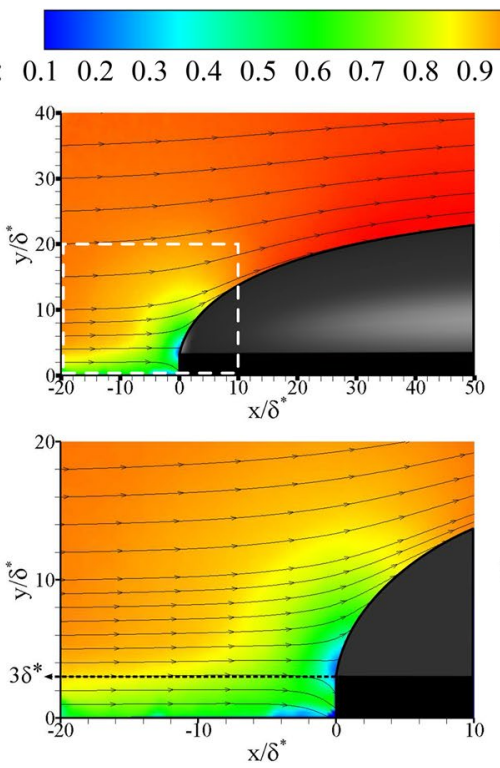

(b) $3 \delta^{*}$
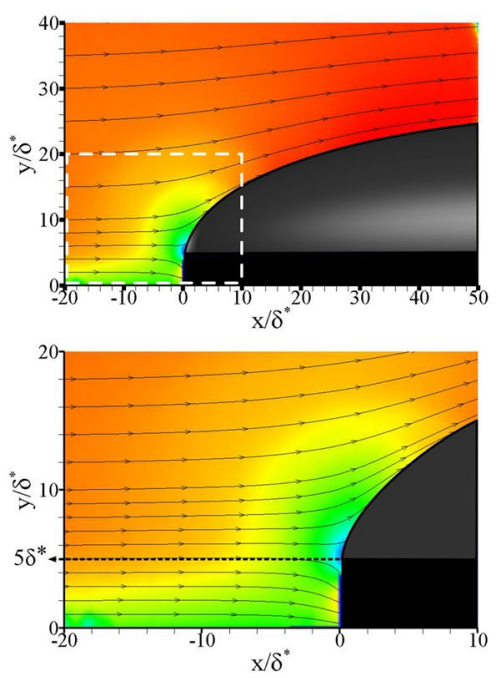

(c) $5 \delta^{*}$

Fig. $10 V_{\mathrm{mag}} / U$ sPIV at the centreline of fuselage nose at $\alpha=0^{\circ}$ (dashed box in upper images illustrates location of enhanced view shown in lower images)

As the flow approaches the fuselage nose $\left(x / \delta^{*}=0\right)$, a point of inflection in the velocity profiles develops which corresponds to the stand-off gap height. This is shown in Fig. $11 \mathrm{~b}, \mathrm{c}$, illustrating the velocity profiles at $x / \delta^{*}=-10$ and $x / \delta^{*}=-5$, respectively. With increasing stand-off height, the approaching airflow below the stand-off gap height is drawn more strongly downstream towards the gap; the flow above the stand-off gap experiences a greater velocity deficit moving over the fuselage nose. The velocity profile immediately upstream of the fuselage nose at $x / \delta^{*}=-0.5$ (Fig. 11d) shows the peak velocity deficit indicating the stagnation point and the jet-like profile of the flow entering the stand-off gap. As the gap further increases, the respective stagnation point moves closer to the symmetry plane and the flow velocity into the gap increases at $4 \delta^{*}$ and $5 \delta^{*}$, this resulting in an excess velocity relative to the empty tunnel boundary layer. As previously mentioned, the lower stand-off height causes an upward deflection of the flow, this is seen here to move the stagnation point away from the symmetry plane of the fuselage. At stand-off heights greater than $3 \delta^{*}$, the stagnation point moves towards the symmetry plane. The velocity profiles above the corresponding standoff height are lifted further away from the tunnel wall due to the model's increased distance from the wall.

Acceleration of the flow under the model, shown in Fig. $11 \mathrm{~d}$, is largely attributed to an increase of the $V_{y}$ component of the flow field below the fuselage symmetry plane, as the flow approaches the stand-off gap. The time-averaged normalised $V_{y}$ component of the flow field and streamlines for all stand-off gap heights tested are presented in Fig. 12, with accompanying $V_{y} / U_{f s}$ velocity profiles shown in Fig. 13. It is clear from Fig. 12 that as the stand-off gap height is increased, the incoming boundary layer is deflected/accelerated downward under the model symmetry plane more strongly. The flow over the upper side of the fuselage does not experience significant changes with the stand-off height other than the position of the stagnation point as previously mentioned. The most significant change of the flow field occurs between $2 \delta^{*}$ and $3 \delta^{*}$. At $3 \delta^{*}$, the stand-off gap height at the fuselage nose reaches approximately $40 \%$ of the empty wind tunnel's boundary layer thickness at the same location.

Upstream from the fuselage nose at location $x / \delta^{*}=-10$, the $V_{y} / U_{\mathrm{fs}}$ velocity profile, shown in Fig. 13a, shows that with the model in closer proximity to the tunnel wall, the upward deflection of the tunnel wall boundary layer is increased. Nearer the model at $x / \delta^{*}=-0.5$, Fig. 13b shows a peak downward $V_{y} / U_{f s}$ velocity located centrally to the respective stand-off gap. The $V_{y} / U_{\mathrm{fs}}$ velocity profiles over the top of the model match closely where apparent discrepancies above the model symmetry plane arise due to the model having different mounting heights depending on stand-off gap.

\subsection{Surface flow patterns}

Measurements from sPIV demonstrated the absence of a horseshoe vortex at the nose of the fuselage, and showed the acceleration of the flow under the model at the centre 


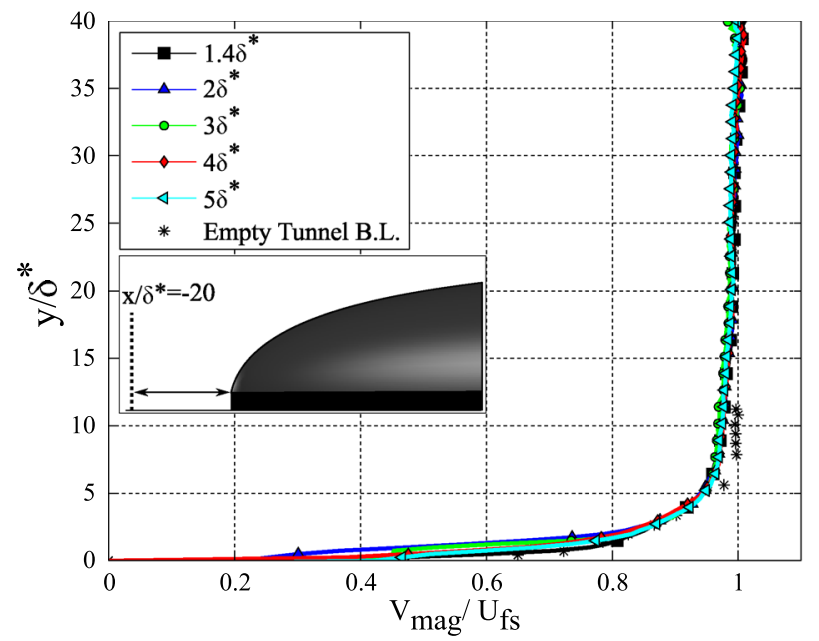

(a) $x / \delta^{*}=-20$ velocity profiles.

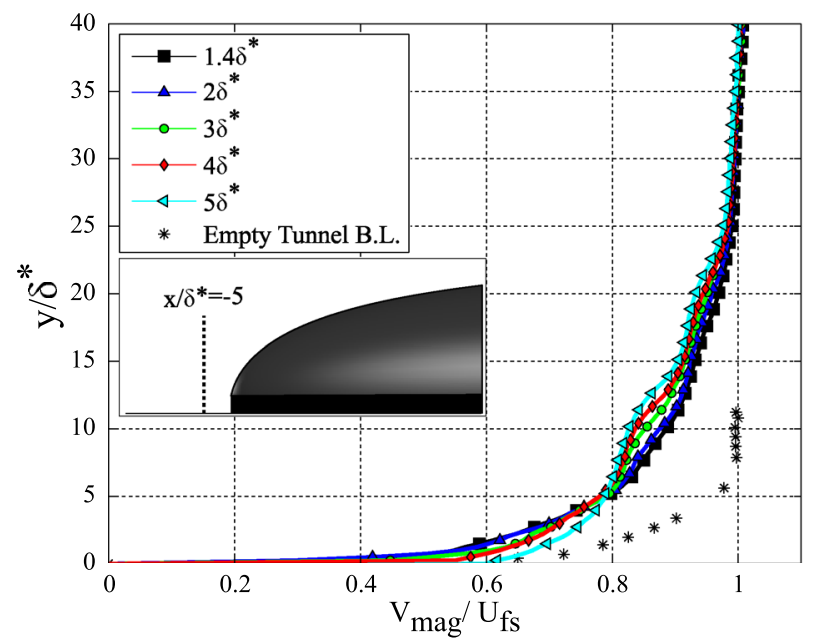

(c) $x / \delta^{*}=-5$ velocity profiles.

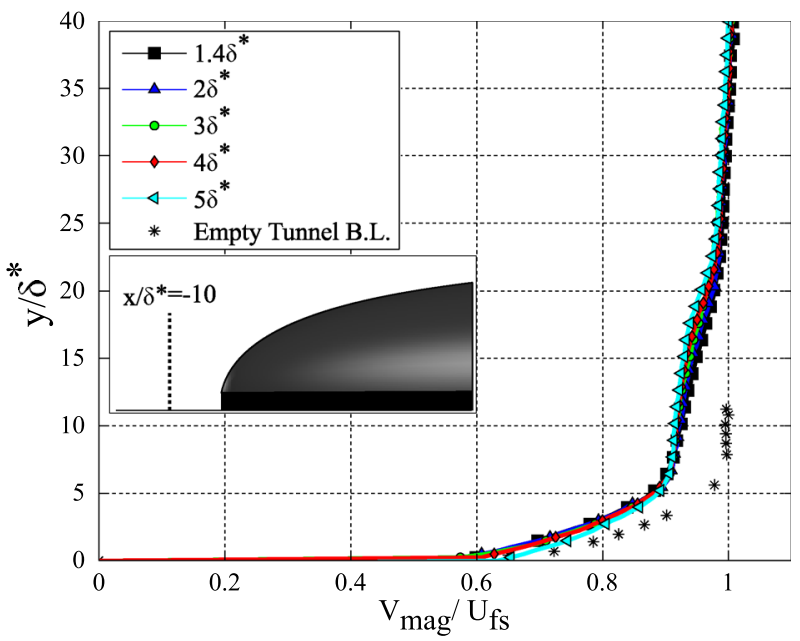

(b) $x / \delta^{*}=-10$ velocity profiles.

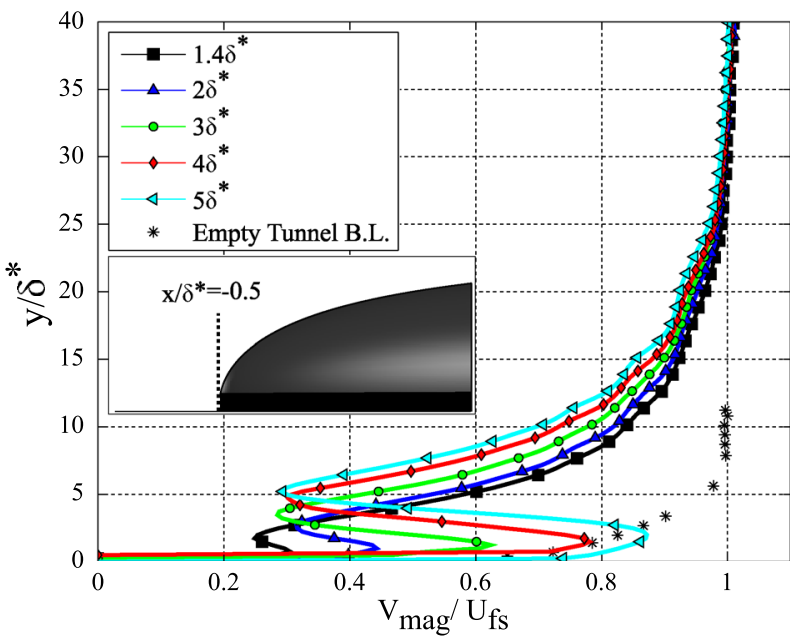

(d) $x / \delta^{*}=-0.5$ velocity profiles.

Fig. 11 Boundary layer profile development at the tunnel wall immediately upstream of fuselage nose/stand-off gap

line for $\alpha=0^{\circ}$. This, however, does not provide information away from the centre line of the fuselage nose or at increased angles of attack. Regions of interest around the model on the floor, along the upper sided of the fuselage, and the wing root region are logistically challenging to investigate with PIV. Consequently, these areas have been qualitatively investigated to enhance the local flow field understanding. Tuft and clay flow visualisation methods highlight regions of flow unsteadiness over the surface and also reveal regions of attached or separated flow, as well as local flow direction. Each method will, however, have intrusive influences on the local flow field, but these effects are assumed negligible.

\subsubsection{Tuft visualisation}

Surface flow visualisation using tufts methods along with the streamlines are presented in Fig. 14. Here, the standard deviation of the fluctuations of the tufts with the model at three stand-off heights $\left(1.4 \delta^{*}, 3 \delta^{*}\right.$, and, $\left.5 \delta^{*}\right)$, at two angles of attack $\left(\alpha=0^{\circ}\right.$ and $\left.14^{\circ}\right)$ is shown. The model geometry is outlined by the dashed lines and free-stream flow direction is shown; the pitch axis is also indicated. The tufts were attached to the tunnel floor, fuselage mid-body/tail, and the wing root. Regions observed to have increased unsteadiness have been highlighted both on the model and floor where the bounding solid lines indicate local shear flows. 


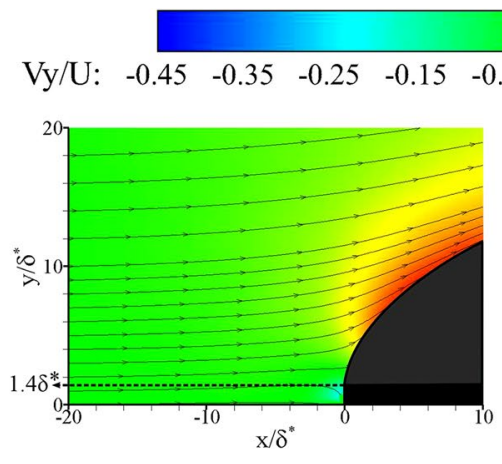

(a) $1.4 \delta^{*}$

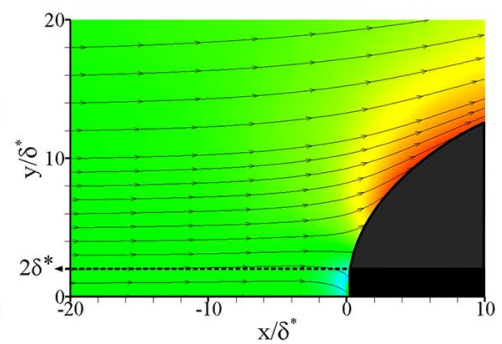

(b)

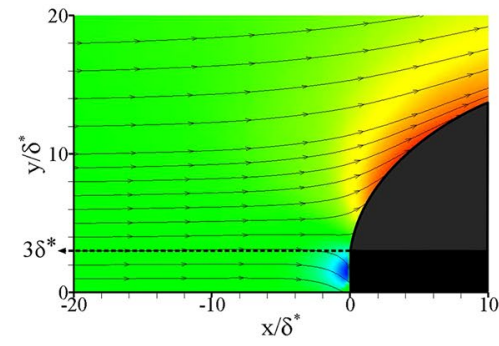

(c) $3 \delta^{*}$

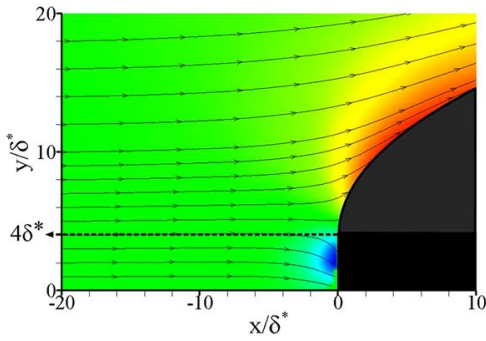

(d) $4 \delta^{*}$



(e) $5 \delta^{*}$

Fig. $12 V y / U$ sPIV at the centreline of fuselage nose at $\alpha=0^{\circ}$

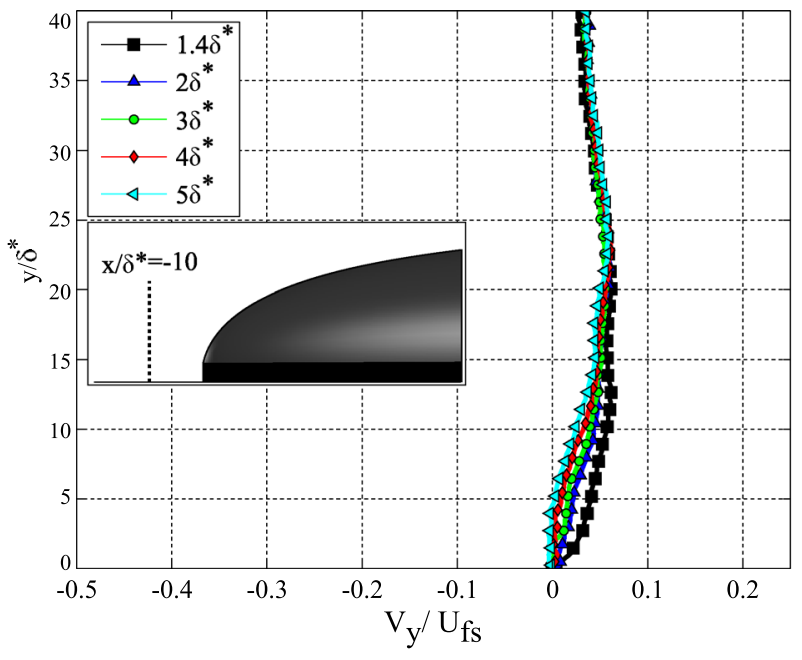

(a) $x / \delta^{*}=-10 \mathrm{y}$-velocity profiles.

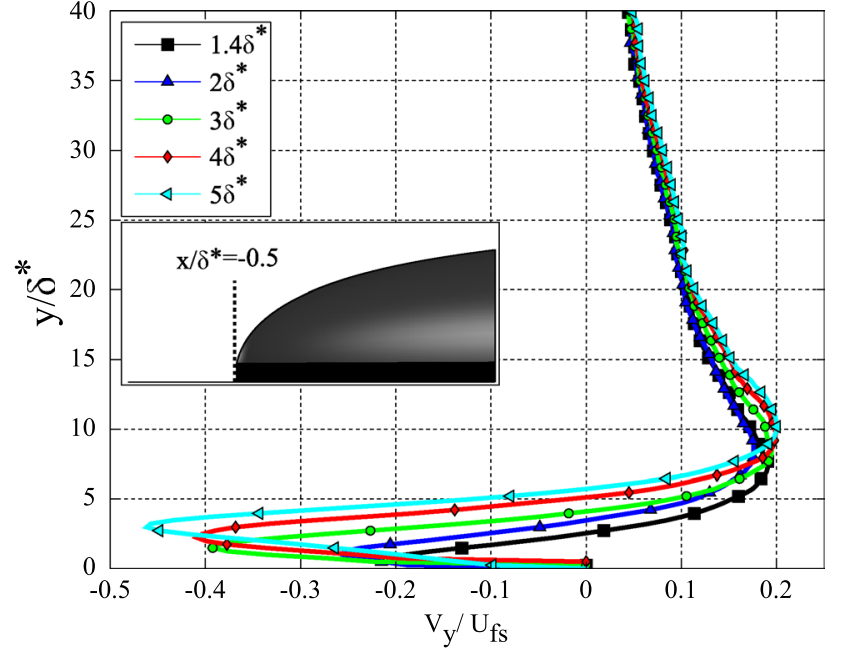

(b) $x / \delta^{*}=-0.5 \mathrm{y}$-velocity profiles.

Fig. $13 V y / U_{f s}$ velocity profiles at the tunnel wall immediately upstream of fuselage nose/stand-off gap

At $\alpha=0^{\circ}$, the stand-off gap is increased from $1.4 \delta^{*}$, to $3 \delta^{*}$, to $5 \delta^{*}$ (Fig. 14a, c, e, respectively). The observed region of tuft activity over the fuselage is seen to reduce, moving aft towards the tail section. Instability in the flow over the entire fuselage is seen to reduce with increasing stand-off height, but the general shape of the disturbance region at $\alpha=0^{\circ}$ does not change with stand-off height. The disturbance patterns on the floor become narrower towards the nose of the fuselage but diffuse away from the fuselage moving downstream, with the outward rate of growth accelerating aft of 




(a) $\alpha=0$, stand-off height $=1.4 \delta^{*}$

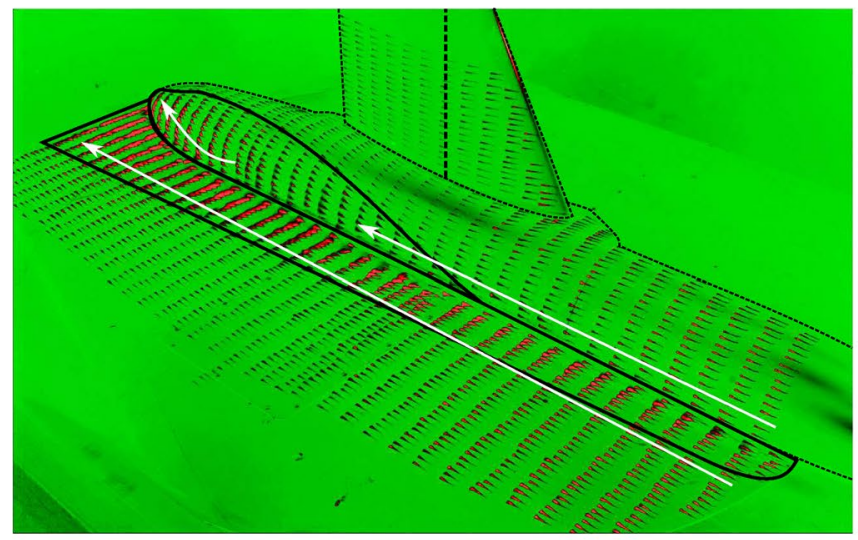

(c) $\alpha=0$, stand-off height $=3 \delta^{*}$

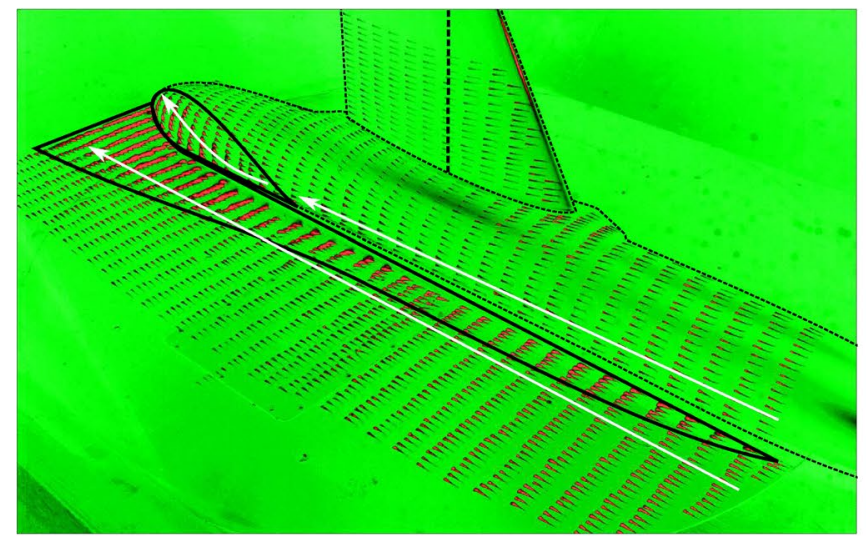

(e) $\alpha=0$, stand-off height $=5 \delta^{*}$

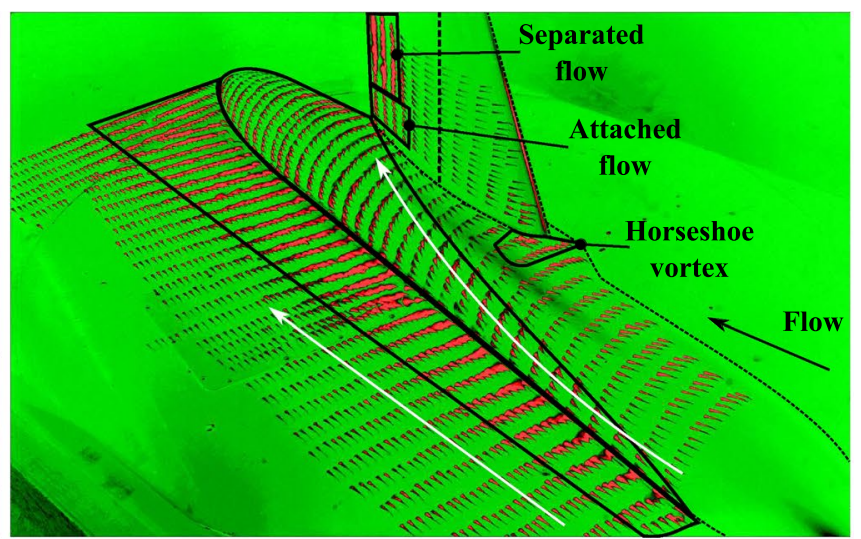

(b) $\alpha=14$, stand-off height $=1.4 \delta^{*}$



(d) $\quad \alpha=14$, stand-off height $=3 \delta^{*}$

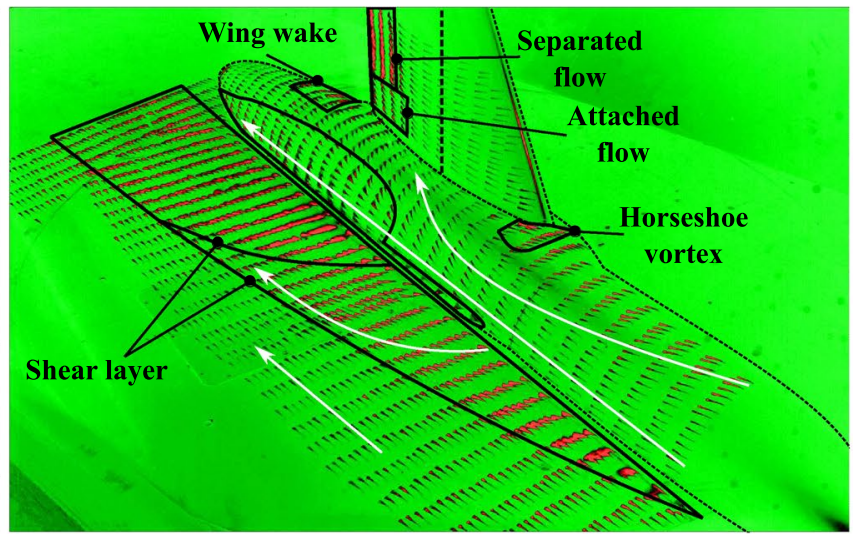

(f) $\alpha=14$, stand-off height $=5 \delta^{*}$

Fig. 14 Qualitative visualisation of the standard deviation in tuft fluctuations for stand-off gap heights $1.4 \delta^{*}, 3 \delta^{*}$, and $5 \delta^{*}$ at $\alpha=0^{\circ}$, and $14^{\circ}$

the pitch axis. Downstream of the pitch axis, activity in the tuft fluctuations increases for each stand-off height. The results indicate that the wake of the shaft under the fuselage introduces a turbulent secondary flow exciting the tufts in all cases. The fluctuation of the tufts on and around the wing root appear independent of stand-off height. This is in agreement with the sPIV, results in Fig. 10, showing the absence of a horseshoe vortex for all stand-off cases; this is known to be the primary influence to alter flow structures at the wing root. 
At $\alpha=14^{\circ}$, as the stand-off gap is increased from $1.4 \delta^{*}$, to $3 \delta^{*}$, to $5 \delta^{*}$ (Fig. $14 \mathrm{~b}, \mathrm{~d}$, f, respectively), it is seen that the region of high fluctuations over the fuselage reduces in size as the stand-off gap is increased. However, unlike the cases at $\alpha=0^{\circ}$ (see Fig. 14a, c, e), the shape of the region and patterns in tuft activity do change with standoff height. The change flow structure arises as secondary flows from between the model's symmetry plane and the tunnel floor becomes more influential as the model's angle of attack is increased. Inspection of the tufts indicating the secondary flow through the stand-off gap suggests that the cross flow from under the fuselage, forward of the pitch axis, is more stable than the flow aft of the pitch axis. Furthermore, at the increased angle of attack, the interference detected ahead of the wing root leading edge is attributed to the presence of a horseshoe vortex formation.

For the stand-off height of $1.4 \delta^{*}$ at $\alpha=14^{\circ}$ (Fig. 14b), tuft fluctuations are present over most of the fuselage, enveloping all of the visible fuselage afterbody. The floor shows high tuft activity along the entire length of the fuselage with no clear flow direction, which is indicative of the flow instability. Tuft fluctuations increase immediately downstream of the shaft (the model pitch axis). As the stand-off height is increased to $3 \delta^{*}$ and $5 \delta^{*}$, Fig. $14 \mathrm{~d}$, f, respectively, the cross flow under the nose of the fuselage becomes more stable. This increase in flow stability reduces the tuft oscillations and shows the local flow direction more clearly. After the pitch axis, the wake of the shaft is seen in the increased tuft activity on the floor.
Figure $14 \mathrm{f}$ shows an approximately mirrored disturbance over the floor and fuselage, starting at the pitch axis of the model. At the maximum stand-off height of $5 \delta^{*}$, and high angle of attack, the shaft wake encounters a faster flow and a larger wake emerging from under the model. In addition, there is a bounding shear layer indicated in Fig. 14f between the cross flow under the semi-span model's nose and the shaft wake. The cross flow under the fuselage nose excites the tufts, but the flow direction is still clear. Aft of the pitch axis, where the shaft wake is present, the turbulent wake excites the tufts enough that a definitive local flow direction is not obvious.

With higher angles of attack, and higher stand-off gap height, the shaft's wake encroaches more and more over the upper side of the fuselage's afterbody. However, this increased influence from the shaft's wake is outweighed by the clear reduction of flow instability along the entire length of the fuselage as gap height is increased. Moving the fuselage symmetry plane away from the proximity of the tunnel floor moves the fuselage higher into the wind tunnel floor's turbulent boundary layer. From the sPIV results, it is shown that at stand-off heights $3 \delta^{*}, 4 \delta^{*}$, and $5 \delta^{*}$, the wind tunnel's boundary layer is drawn underneath the model.

\subsubsection{Clay flow visualisation}

Figures 15,16 show the surface flow clay patterns on the tunnel floor around the model at a gap height of $5 \delta^{*}$ at $\alpha=0^{\circ}$ and $14^{\circ}$, respectively. This enables observations of the interaction between the boundary layer flow on the fuselage and
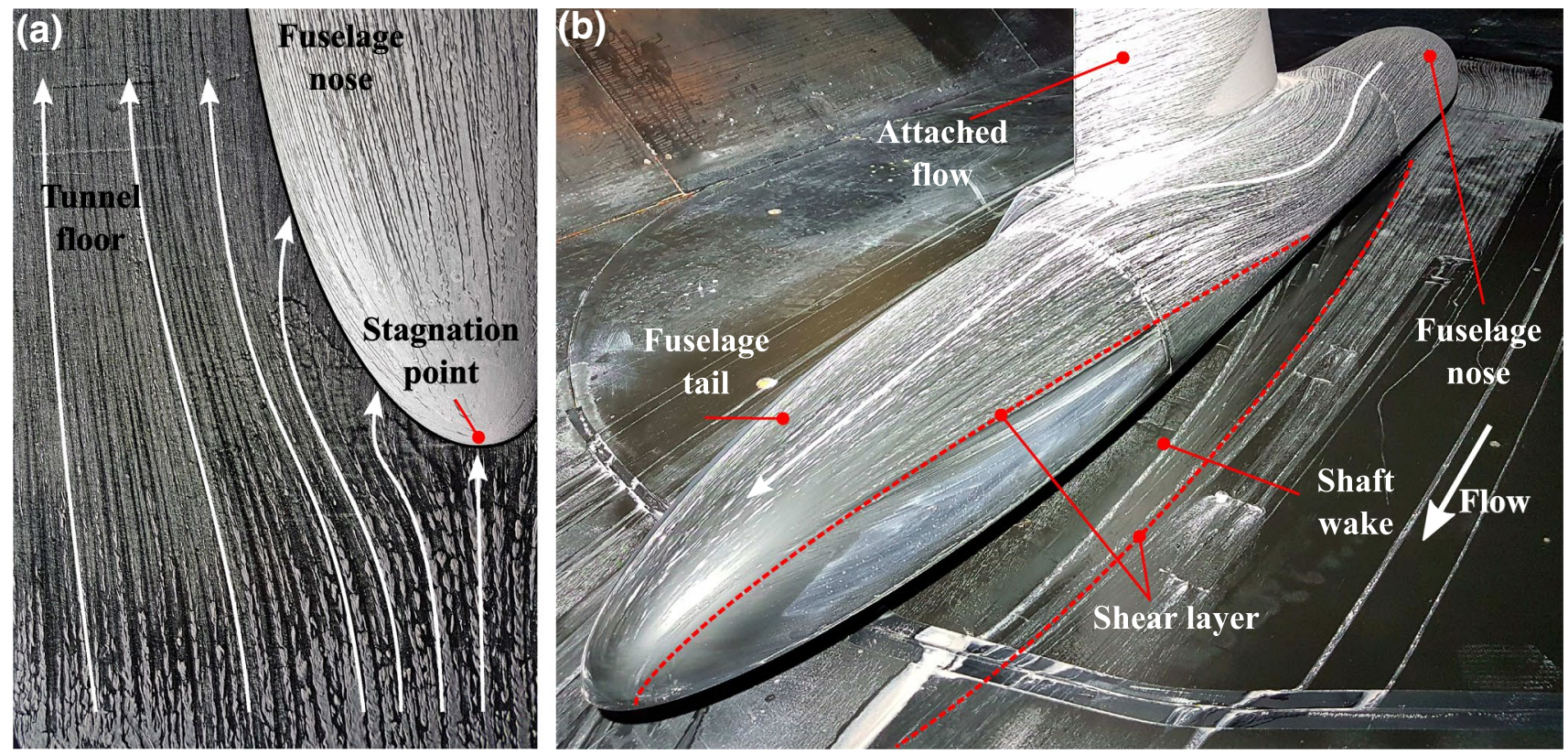

Fig. 15 Clay flow around model at $\alpha=0$ at stand-off height $5 \delta^{*}$; views of fuselage nose (a) and full fuselage, wing root, and floor (b) are shown 

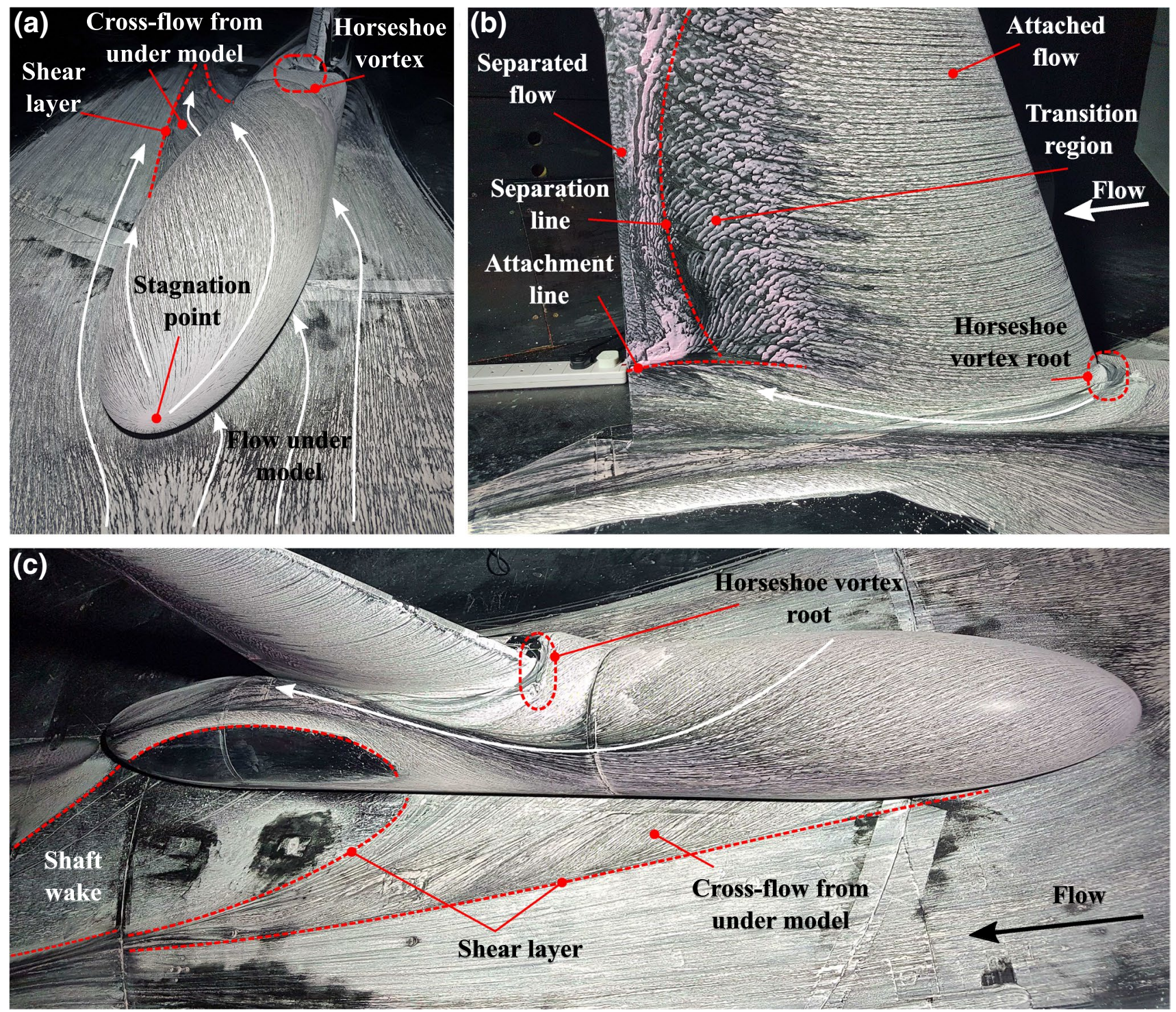

Fig. 16 Clay flow around model at $\alpha=14$ at stand-off height $5 \delta^{*}$; views of fuselage nose (a), wing root (b) and full fuselage, wing root, and floor $(\mathbf{c})$ are shown

the inboard wing area and also how disturbances propagate and develop over the model at this stand-off height. From the sPIV results in Fig. 10, it was evident that the flow accelerates under the model. Without a peniche, there is a velocity gradient at the wall in front of the fuselage nose. Therefore, due to the acceleration of the flow into the gap under the model, the flow does not separate from the wind tunnel wall. Hence, no horseshoe vortex is formed. The effect of this is visualised using the clay flow method at $\alpha=0^{\circ}$ in Fig. 15a. The streamlines formed by the clay around the fuselage nose, in Fig. 15a, show the flow turning and being drawn under the fuselage. This corresponds well to the sPIV data in Fig. 10 illustrating the flow accelerating into the stand-off gap. Figure 15 was conducted under identical conditions as the tuft visualisation in Fig. 14e, and sPIV experiments in Fig. 10c. Comparing the Figs. 15b and 14e, the influence of the shaft wake is visible in both instances. In the tuft visualisations, the shaft wake increases flow instability over the tufts, but in the clay flow, the wake results in 'clean' patches over the floor and fuselage afterbody following the shaft wake's shear layer with the surrounding flow. The clay/ kerosene mixture was pushed around the shaft wake due to the wake's relatively higher static pressure. The overall wake pattern outlined by the clay (Fig. 15b) corresponds closely to regions of heightened tuft fluctuations in Fig. 14e.

Applying the clay mixture to the model at $\alpha=14^{\circ}$ results in the surface flow patterns shown in Fig. 16. The absence of the horseshoe vortex is again illustrated by the clay flow 
patterns, with the flow streamlines being drawn under the model as previously seen at $\alpha=0^{\circ}$ in Fig. 15a. The streamline curvature over the fuselage length is shown in Fig. 16a, $\mathrm{c}$, which is strongly influenced by the induced velocity over the wing root and also the shaft wake. Importantly, the streamlines show an attached flow over the entire fuselage at this high angle of attack.

Figure 16b shows flow patterns arising from the induced velocity over the wing root. Due to the tuft visualisations at the wing root and consistent absence of the horseshoe vortex at the fuselage nose, it is expected that the flow over the inboard wing is similar for all stand-off heights. Akin to the tuft visualisations, with comparison made to Fig. 14f, the horseshoe vortex ahead of the wing root leading edge and the regions of separated and attached flow towards the wing trailing edge is observed. The wing leading edge horseshoe vortex is responsible for the flow reattachment toward the wing root in Fig. 16b with the reattachment and separation lines highlighted. Attached flow over the inboard section of the wing and transitional flow, suggesting boundary layer thickening, is also indicated. It is necessary to bring attention to the possible influence of gravity on the surface patterns in this region. The boundary layer thickens and develops in the transitional region, indicative of the local wall shear stresses weakening, at which point gravitational forces may begin to dominate (note that wing is mounted vertically) and draw the clay mixture down toward the fuselage. However, from experimental observations and the development of the surface streamlines with time, it is assumed that forces due to gravity are negligible, and that fluid dynamic shear forces from the flow on the clay remain dominant.

As the angle of attack increases, the shaft wake impinges more onto the top side of the fuselage, the effect of this is clear when comparing Figs. 15b and 16c. However, from the tuft visualisations, it is understood that the instabilities over the entire fuselage would be greater at lower gap heights.

\section{Conclusions}

An experimental investigation has been performed on a semi-span wind tunnel model at several stand-off gap heights, without a peniche, at $R e=1.5 \times 10^{6}$ based on the wing mean aerodynamic chord. Force platform data were used to evaluate aerodynamic coefficients, while sPIV and surface flow visualisations provided information on how the local flow field around the model varied with both stand-off gap height and angle of attack. Several stand-off gap heights were tested, based on the empty wind tunnel boundary layer displacement thickness $\left(\delta^{*}\right)$ of $1.4 \delta^{*}$ to $5 \delta^{*}$.

The aerodynamic behaviour and flow characterisation suggest that semi-span model testing without a peniche leads to more stable results/flow field than what would be achieved with a 2D-peniche. It is observed that the lift and pitching moment coefficients decrease as the stand-off gap becomes higher, but drag increases. Over the linear lift region of the model $(-5 \leq \alpha \leq+7)$, deviation of the aerodynamic coefficients is a function of the distance between the fuselage symmetry plane and the tunnel wall, and not a function of the angle of attack. Raising the stand-off gap from $1.4 \delta^{*}$ to $5 \delta^{*}$ resulted in an approximately constant offset increase in total drag by $\Delta C_{\mathrm{D}}=+0.0072$ (approximately a $26 \%$ increase). The lift-curve slope decreased with the stand-off gap in a linear fashion; this trend is independent of the Reynolds number.

These results suggest that removal of the peniche leads to a more robust model set-up which operates in a more stable flow field insensitive to changes in angle of attack. It is also, relatively, less sensitive to the stand-off height. A peniche extrusion generates a vortex in front of the fuselage at the wall juncture, which in turn is known to influence the flow over the fuselage and inboard wing. By removing the peniche and leaving a gap between the fuselage symmetry plane and the wall, deviations in aerodynamic behaviours were found independent of the angle of attack, especially prior to separation onset at the wing. Dynamic loading (from wing vibration) and frequency analysis show that the model is, perhaps, more vulnerable to forced vibration due to the absence of the peniche, resulting in less structural support. However, to install a model of this type with a peniche, a force platform would drastically increase model complexity to prevent fouling on the tunnel wall.

With higher angles of attack, and higher stand-off gaps, the mounting shaft's wake encroaches more and more over the fuselage's afterbody. However, this increased influence of the shaft's wake is outweighed by the clear reduction of flow instabilities along the entire length of the fuselage as the stand-off gap is increased. At a stand-off gap height of $4 \delta^{*}$ to $5 \delta^{*}$, the tunnel boundary layer is drawn underneath the model, this moves the stagnation point closer to the symmetry plane of the fuselage. This is representative of the flow field expected around an equivalent full-span model.

Acknowledgements The authors would like to express our gratitude and appreciation to the low-speed National Wind Tunnel Facility (EPSRC Grant no.: EP/L024888/1) at the University of Glasgow. Great appreciation must also be registered for Dr. Richard Green, whose support and technical expertise have been invaluable. In addition, the authors would like to thank Zephyr Business Services and Royal Aeronautical Society for funding this work.

Open Access This article is distributed under the terms of the Creative Commons Attribution 4.0 International License (http://creat ivecommons.org/licenses/by/4.0/), which permits unrestricted use, distribution, and reproduction in any medium, provided you give appropriate credit to the original author(s) and the source, provide a link to the Creative Commons license, and indicate if changes were made. 


\section{References}

Anderson JD (2001) Fundmentals of aerodynamics, 3rd edn. McGrawHill, New York

Barlow JB, Rae WH, Pope A (1999) Low-speed wind tunnel testing, 3rd edn. Wiley

Doerffer Piotr, Szulc Oskar (2006) High-lift behaviour of half-models at flight reynolds numbers. Task Q 10(2):191-206

Eder S, Hufnagel K, Tropea C (2006) Semi-span testing in wind tunnels. In: 25th International Congress of the Aeronautical Sciences: ICAS, pp 1-9

Eliasson P (2008) Investigation of a half-model high-lift configuration in a wind tunnel. J Aircr 45(1):29-37

Gatlin GM, McGhee RJ (1996) Study of semi-span model testing techniques. In: NASA-TM-111454

Gatlin GM, Parker PA, Owens LR (2001) Development of a semi-span test capability at the national transonic facility. In: 39th AIAA Aerospace Sciences Meeting and Exhibit

Kafyeke F, Pépin F, Kho C (2002) Development of high-lift systems for the Bombardier CRJ-700. In: ICAS 2002 Congress, pp 1-10

Keane RD, Adrian RJ (1992) Theory of cross-correlation analysis of PIV images. Appl Sci Res 49:191-215

Kuo HC, Lin DC (1996) Non-uniform recovery of vortex breakdown over delta wing in response to blowing along vortex core. Exp Fluids 22:33-44

Lee T, Su YY (2012) Aerodynamic performance of a wing with a deflected tip-mounted reverse half-delta wing. Exp Fluids 53:1221-1232

Lynch FT (1992) Experimental necessities for subsonic transport configuration development. AIAA Paper 92-0158
Malik A, Render PM (2010) Use of wall suction in half model wind tunnel testing. In: 28th Applied Aerodynamics Conference, Chilcago, Ill, USA

Millholen WE II, Chokani N (1996) Effect of side boundary layer on transonic flow over a wing. J Aircr 33(6):1115-1122

Millholen WE II, Chokani N, McGhee RJ (1996) Development of semispan model test. Techniques 33(6):47065

Nguyen N, Ting E, Lebofsky S (2015) Aeroelastic analysis of a flexible wing wind tunnel model with variable camber continuous trailing edge flap design. 56th AIAA/ASCE/AHS/ASC Structures, Structural Dynamics, and Materials Conference, pp 1-28

Scarano F (2008) Topics in applied physics, vol 112. ISBN 9783540735274

Silva WA, Iii BP, Florance JR, Sanetrik MD, Wieseman CD, Stevens WL, Funk CJ, Coulson DA (2012) An overview of the semi-span super-sonic transport (S4T) wind-tunnel model program. 53rd AIAA/ASME/ASCE/ASC Structures, Structural Dynamics and Materials Conference, (AIAA 2012-1552), pp 1-18

Ujang MI, Mat S, Perumal K, Mohd-Nasir MN (2016) Experimental study of UTM-LST generic half model transport aircraft. AEROTECH VI-Innovation in Aerospace Engineering and Technology, p 152

Viehweger G, Ewald B (1994) Half model testing in cologne cryogenic tunnel (KKK). AIAA Paper 94-2511

Yokokawa Y, Murayama M, Uchida H, Tanaka K (2010) Aerodynamic influence of a half-span model installation for high-lift configuration experiment. In: 48th AIAA Aerospace Sciences Meeting Including the New Horizons Forum and Aerospace Exposition, AIAA 2010-684, number January, Orlando, Florida 